\title{
Pembrolizumab in a Patient With Heavily Pre-Treated Squamous Cell Thymic Carcinoma and Cardiac Impairment: A Case Report and Literature Review
}

\begin{abstract}
Alessandro Cafaro ${ }^{1}$, Alberto Bongiovanni ${ }^{2 *}$, Valentina Di lorio ${ }^{1}$, Devil Oboldi ${ }^{3}$, Carla Masini ${ }^{1}$ and Toni Ibrahim ${ }^{2}$

1 Oncological Pharmacy Unit, Istituto Scientifico Romagnolo per lo Studio e la Cura dei Tumori (IRST) IRCCS, Meldola, Italy, ${ }^{2}$ Osteoncology and Rare Tumors Center, Istituto Scientifico Romagnolo per lo Studio e la Cura dei Tumori (IRST) IRCCS, Meldola, Italy, ${ }^{3}$ Radiology Unit, Istituto Scientifico Romagnolo per lo Studio e la Cura dei Tumori (IRST) IRCCS, Meldola, Italy
\end{abstract}

Immunotherapy directed at the programmed cell death-1 receptor (PD-1) or its ligand PD-L1 has proven effective in solid malignancies such as melanoma, non-small cell

OPEN ACCESS

Edited by:

Jessica Desiree Menis,

Veneto Institute of Oncology

(IRCCS), Italy

Reviewed by:

Lizza E. L. Hendriks,

Maastricht University Medical

Centre, Netherlands

Sara Pilotto,

University of Verona, Italy

${ }^{*}$ Correspondence:

Alberto Bongiovanni

alberto.bongiovanni@irst.emr.it

Specialty section:

This article was submitted to Pharmacology of Anti-Cancer Drugs,

a section of the journal

Frontiers in Oncology

Received: 20 May 2020

Accepted: 10 July 2020

Published: 18 August 2020

Citation:

Cafaro A, Bongiovanni A, Di lorio V, Oboldi D, Masini C and Ibrahim T (2020) Pembrolizumab in a Patient With Heavily Pre-Treated Squamous Cell Thymic Carcinoma and Cardiac Impairment: A Case Report and

Literature Review.

Front. Oncol. 10:1478.

doi: 10.3389/fonc.2020.01478 lung cancer (NSCLC), urothelial cancer, renal cell carcinoma, and head and neck cancer. Compared with cytotoxic chemotherapy, radiotherapy, or molecular targeted agents, immunotherapy is an innovative strategy for treating malignancies, with durable clinical responses and manageable adverse events. Thymic carcinomas are extremely rare, constituting only $0.06 \%$ of all malignancies, are much more aggressive tumours than thymomas and have a worse prognosis. Nowadays, first line platinum-based chemotherapy for metastatic tumours are the cornerstone of treatment. However, the results of further therapeutic lines for metastatic or relapsed thymic carcinoma are unsatisfactory, with no regimen showing a consistent benefit. Moreover, the rarity of these tumours makes it difficult to carry out clinical trials. Herein we report a remarkable result of 1 year stable disease with good quality of life and no side effects obtained from the use of immunotherapy with pembrolizumab in a case of heavily pre-treated squamous cell thymic carcinoma and cardiac impairment. We also include a literature review of clinical trials on PD-1/PD-L1 inhibitors for the treatment of thymic epithelial cancers, taking a close look at cardiac toxicity.

Keywords: pembrolizumab, immune checkpoint inhibitors, thymic cancer, squamous cell, PD-1, PD-L1, cardiotoxicity, case report

\section{INTRODUCTION}

Based on the RARECARE project definition, thymoma, and thymic carcinomas defined as Thymic Epithelial Tumours [TETs] are rare cancers (1). The annual incidence of TETs has been estimated as 1.3-3.2 cases per million worldwide and thymic carcinomas are even rarer, accounting for only $0.06 \%$ of all thymic neoplasms (2). Thymic carcinoma (TC) can be histologically classified into several subtypes including squamous, basaloid, mucoepidermoid, sarcomatoid, small cell, adenocarcinoma lymphoepithelial-like, clear cell, and undifferentiated of which the squamous cell subtype is the most common and represents $73-79 \%$ of the total cases (3-5). TC is an aggressive 
tumour with a poorer prognosis than thymoma (6). Fiveyear survival for locally advanced TC is $36 \%$, decreasing to $24 \%$ for metastatic disease (7). Surgical resection of the primary tumour remains the cornerstone of therapy for earlystage disease. In advanced or recurrent TETs, a multimodality approach including surgery, chemotherapy, and radiotherapy is required (8).

Platinum-based chemotherapy remains the gold-standard in the first-line setting. The most frequently used treatment is the platinum-based combination, but fewer than $50 \%$ of patients respond $(8,9)$. Randomized prospective clinical trials are lacking due to the rarity of these tumours, making it important to discuss therapeutic strategies in a multidisciplinary setting. After progression on platinum-based therapy there are limited indications for second-line treatments like sunitinib and everolimus, with data mainly extrapolated from small, single-arm, phase II clinical trials, or retrospective observational studies. VEGF plays a key role in tumour angiogenesis and VEGFR2 and PDGFR- $\alpha$ are known to be activated in thymic carcinoma (10). The activity of sunitinib malate and Lenvatinib, two multi-targeted kinase inhibitors were tested in two phase 2 trials, in which 26 and $38 \%$ of patients with thymic carcinoma achieved a partial response, respectively $(11,12)$. Anthracyclinebased chemotherapy is recommended also by the National Comprehensive Cancer Network (NCCN) guidelines but the response rate is similar to that of other regimens and the problem of the best treatment in this setting remains open to debate $(11,13-15)$. Immuno-oncology (IO) is changing the therapeutic landscape of thoracic malignancies, including TETs, although results are not still not conclusive $(16,17)$. Secondline pembrolizumab has shown promising activity, Giaccone et al. reporting a median response rate of $22.5 \%$ (range 10.8 $38.5 \%$ ) and a median PFS of 4 months (18). However, such findings require confirmation in large prospective clinical trials. Furthermore, PD-L1 expression has been reported in $36-75 \%$ of TCs but its prognostic and predictive value remain controversial. Another issue in therapeutic decision-making relating mainly to the choice of IO is the presence of co-morbidities, especially those of a cardiological nature. The incidence of cardiac complications in patients with TC seems to be associated with the use of anthracycline-based chemotherapy and mediastinal radiotherapy (19). Furthermore, myocarditis has been reported in $5-9 \%$ of TET patients treated with pembrolizumab $(18,20)$. Herein we describe the case of a heavily pre-treated patient with squamous cell TC and left ventricular dysfunction in which the administration of the PD-1 antibody pembrolizumab obtained disease control lasting more than a year, without cardiac failure.

\section{CASE DESCRIPTION}

In September 2016a 57-year-old man was diagnosed with a squamous cell TC (diameter $3.6 \mathrm{~cm}$ ) with involvement of the sternal manubrium and bone metastases (sternum and ribs) (stage IVa according to the Masaoka-Koga system) (21). Eastern Cooperative Oncology Group performance status (ECOG PS) was 0 . The patient was a never-smoker with no history of autoimmune disorders. He had mild obesity (BMI: $37 \mathrm{~kg} / \mathrm{m}^{2}$ ) as a co-morbidity. From October 2016 to February 2017, six courses of 3-weekly first-line chemotherapy with carboplatin and paclitaxel were administered. After three cycles the patient obtained a partial response (PR) according to RECIST (Response Evaluation Criteria in Solis Tumours) 1.1, confirmed at the end of the sixth cycle (22). Sequential radiotherapy was performed on the mediastinal mass (60 Gy in 30 fractions). Four months after the end of the first-line treatment an ${ }^{18}$ FDG-PET/CT (18fluorodeoxy-glucose positron emission tomography-computed tomography) scan showed areas of increased uptake in the 6 th and 7 th right ribs and progressive disease in the sternal body according to MD Anderson criteria (23). From July to December 2017 the patient underwent second-line therapy with gemcitabine, but an ${ }^{18} \mathrm{FDG}-\mathrm{PET} / \mathrm{CT}$ revealed progression of the primary mediastinal mass, new lesions in the posterior arch of the 6th right rib and pulmonary hilar lymph node involvement. A CT scan confirmed these findings. On January 2018 the patient was referred to our institute for a second opinion. After a multidisciplinary discussion, it was decided to perform a 68 Gallium (68Ga)-DOTATOC-PET/CT, which showed very slight somatostatin receptor uptake by both the primary and metastatic lesions (Figure 1). This excluded the patient from being enrolled in a phase II trial for tumours expressing somatostatin receptors type 2 (sstr2) with Luthatera ${ }^{\circledR}$ peptide receptor radionuclide therapy (NCT03454763). Given the previous treatment received and the good ECOG PS, third-line chemotherapy with a modified ADOC (mADOC) scheme (doxorubicin $40 \mathrm{mg} / \mathrm{m}^{2}$, vincristine $0.60 \mathrm{mg} / \mathrm{m}^{2}$, cyclophosphamide $700 \mathrm{mg} / \mathrm{m}^{2}$ ) without cisplatin was started in February 2018, obtaining a PR after the third cycle. The cardiac evaluation performed before treatment had shown a normal heart function with a left ventricular heart fraction (LVHF) of 61\%. In July 2018 there was a reduction in LVHF (49\%) with mild symptoms (class 2 according the New York Heart Association Classification) caused by the previous treatment and general conditions, and mADOC treatment was stopped. Echocardiography showed concentric left ventricular hypertrophy, cardiac hypokinesia and a further decrease in LVHF (43\%). In November 2018 a CT scan documented further disease progression with the appearance of multiple bilateral pleural and pulmonary lesions and suspected liver metastases. Molecular evaluation of PD-L1 expression and other mutations was requested but not performed because of insufficient bioptic tissue. After a multidisciplinary team discussion with the institute's cardio-oncologist, the patient was deemed unamenable to treatment with sunitinib given the known cardiotoxicity of tyrosine kinase inhibitors (TKIs) and the risks associated with its use in a patient with the aforementioned comorbidity. In February 2019, given the good clinical conditions and the relatively young age of the patient, it was decided to start offlabel fourth-line treatment with pembrolizumab (200 mg every 3 weeks) (approved by the Italian Medicines Agency in accordance with Art. 48, Leg. 326/2003) and monitor cardiac side-effects. The beta 1-selective-adrenoceptor blocking agent bisoprolol and an angiotensin-converting enzyme inhibitor (ramipril) were added by the cardio-oncologist. The patient underwent 18 courses of 


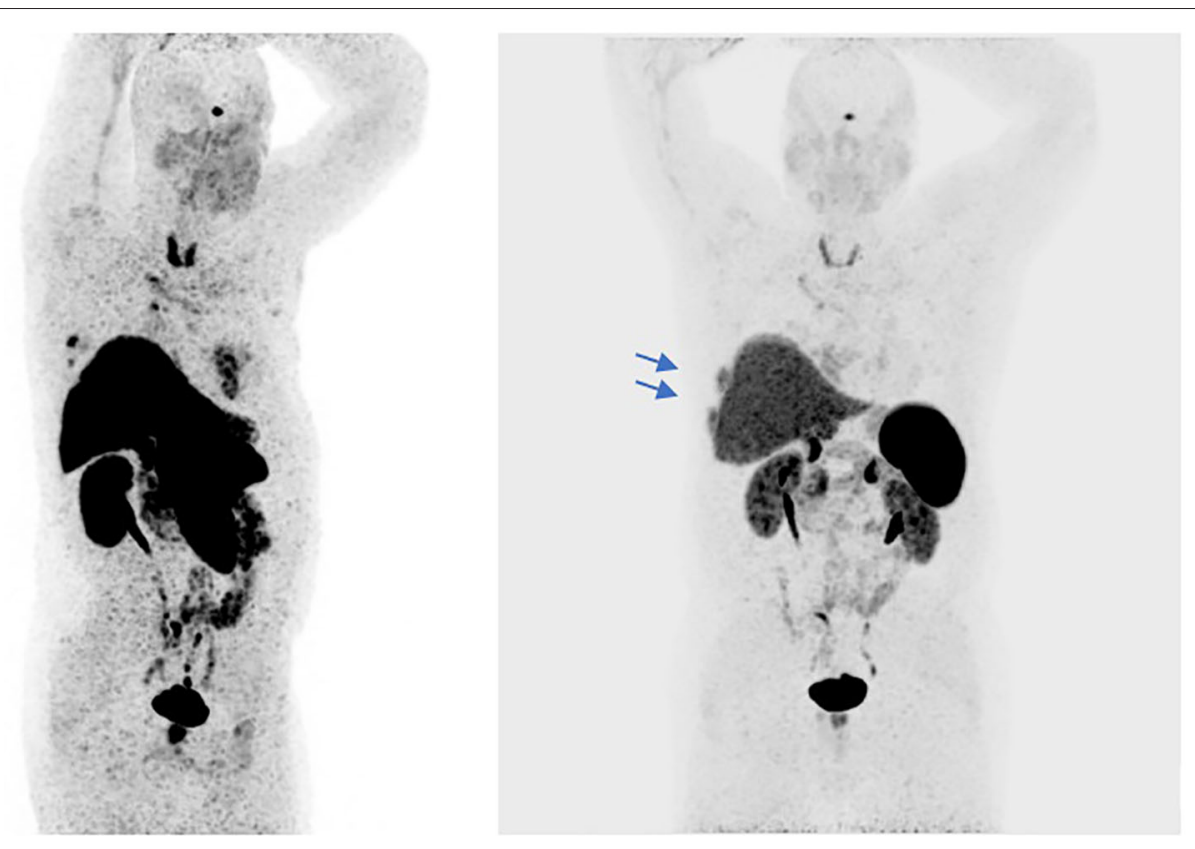

FIGURE 1 | The ${ }^{68} \mathrm{Ga}$-PET/CT shows areas of slight somatostatin receptor uptake in the right subpleural paracardiac area, standardized uptake value (SUVmax = 5.2), and some pre-vascular (SUVmax = 2.3), retrosternal (SUVmax = 3.2), Barety lodge (SUVmax = 3), and right peribronchial (SUVmax = 5.4) node uptake (red arrows). Some areas in the dorsal segment of the upper lobe of the right lung and in middle lobe of the right lung showed no uptake. Slight uptake of the radiopharmaceutical product in the sternal body (SUVmax $=5.9$ ) and the VI and VII right ribs (SUVmax $=8.6)$ (blue arrows).

three-weekly pembrolizumab, obtaining stable disease for more than 1 year (Figure 2).

During this period, cardiac function was monitored closely (echocardiography and blood tests) to detect even the slightest sign of worsening heart failure. Levels of myosin light chain troponin, N-terminal prohormone of brain natriuretic peptide (NT-proBNP), lactate dehydrogenase and creatine phosphokinase were normal and monitored over time. No signs of heart damage were detected and no adverse events were reported. In March 2020 the patient was hospitalized for dyspnoea, a CT scan showing bilateral pleural effusion. More than 2,000 $\mathrm{mL}$ of exudative fluid was drained by thoracentesis and cytology was positive for TC cells. Another CT performed in April confirmed disease progression according to immunerelated RECIST, with an increase in the size of the primary tumour and pleural localizations. Echocardiography of April 2020 revealed an improvement in cardiac function, with normal LVHF (62\%). It was decided to stop pembrolizumab and after a multidisciplinary discussion the patient started treatment with sunitinib (Figure 3).

\section{DISCUSSION}

Several studies have investigated the role of IO in TCs. Although PD-L1 is mainly expressed in cortical and medullary thymic epithelial cells (24), Padda et al. observed that high PD-L1 scores were found more often in TETs than in normal thymus tissue and that its expression was inversely related with clinical outcome
(25). On the basis of these findings, the blockage of the PD1/PD-L1 axis by specific monoclonal antibodies (MAB) could be a promising treatment for TC.

The efficacy of the anti PD-L1 pembrolizumab was evaluated by Giaccone et al. in 40 patients with recurrent TC in progression after at least one line of chemotherapy in a single-arm, single centre, phase II study. Patients had received a median of two previous therapies. Nine (23\%) patients achieved an objective response, one a complete response (CR) and $8 \mathrm{PR}$ and 21 (53\%) stable disease (SD). Medium progression-free survival (PFS) was 4.2 months (95\% CI 2.9-10.3) and was longer in patients with high PD-L1 expression [TPS (tumour proportion score) $\geq 50 \%$ ] than in those with low or no PD-L1 expression (median PFS of 24 months, 95\% CI 5.8-42.3 vs. 2.9 months, 95\% CI 1.74.1). The disease control rate (DCR) was $75 \%$, higher than that seen in Sunitinib study and 3 of 21 patients who obtained a SD maintained this response for more than a year $(11,19)$. A head to head comparison should clarify these findings.

Another phase II trial tested pembrolizumab in 33 patients with TET in whom platinum-based systemic chemotherapy was unsuccessful. The overall response rate was $21 \%$, disease control rate (DCR) 79\%, and median PFS was 6.1 months. High PDL1 levels were confirmed as a significant predictive marker of response to therapy (21). Conversely, Katsuya et al. reported conflicting results in a phase II trial evaluating nivolumab in 15 patients with previously treated TC, six of whom had received more than 3 previous systemic treatments. Notably, no data about PD-L1 TPS were reported. No objective responses were 

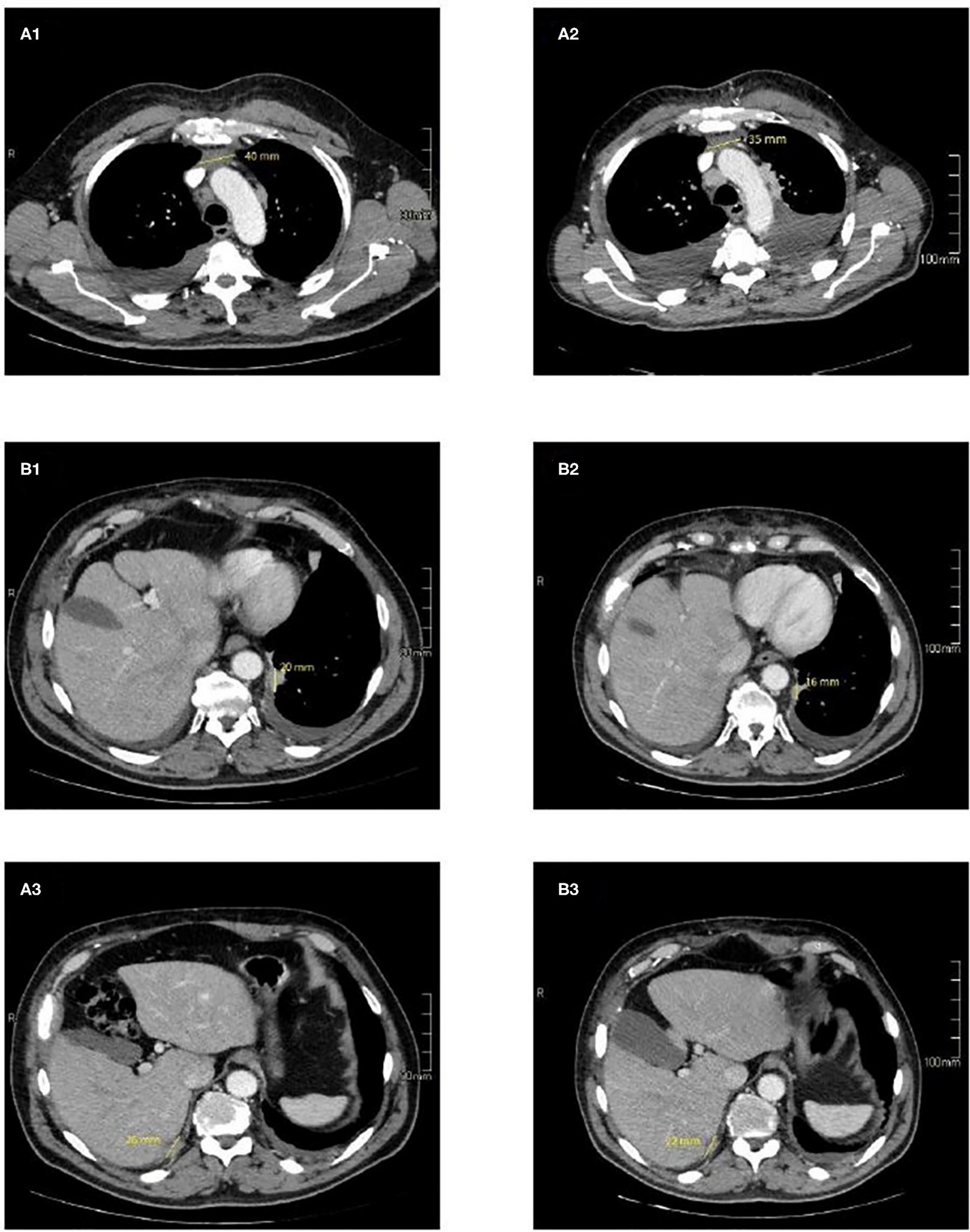

FIGURE 2 | CT imaging of selected target lesions at baseline (A) and during treatment (B). Representative axial CT images of patient shows a slight reduction in size of the primitive tumour, $35 \mathrm{~mm}$ (B1) vs. $40 \mathrm{~mm}$ (A1) (blue arrows) and pleural localizations on the left side $16 \mathrm{~mm}$ (B2) vs. $20 \mathrm{~mm}$ (A2) (yellow arrows), and right side $22 \mathrm{~mm}$ (B3) vs. $26 \mathrm{~mm}$ (A3) (red arrows). 


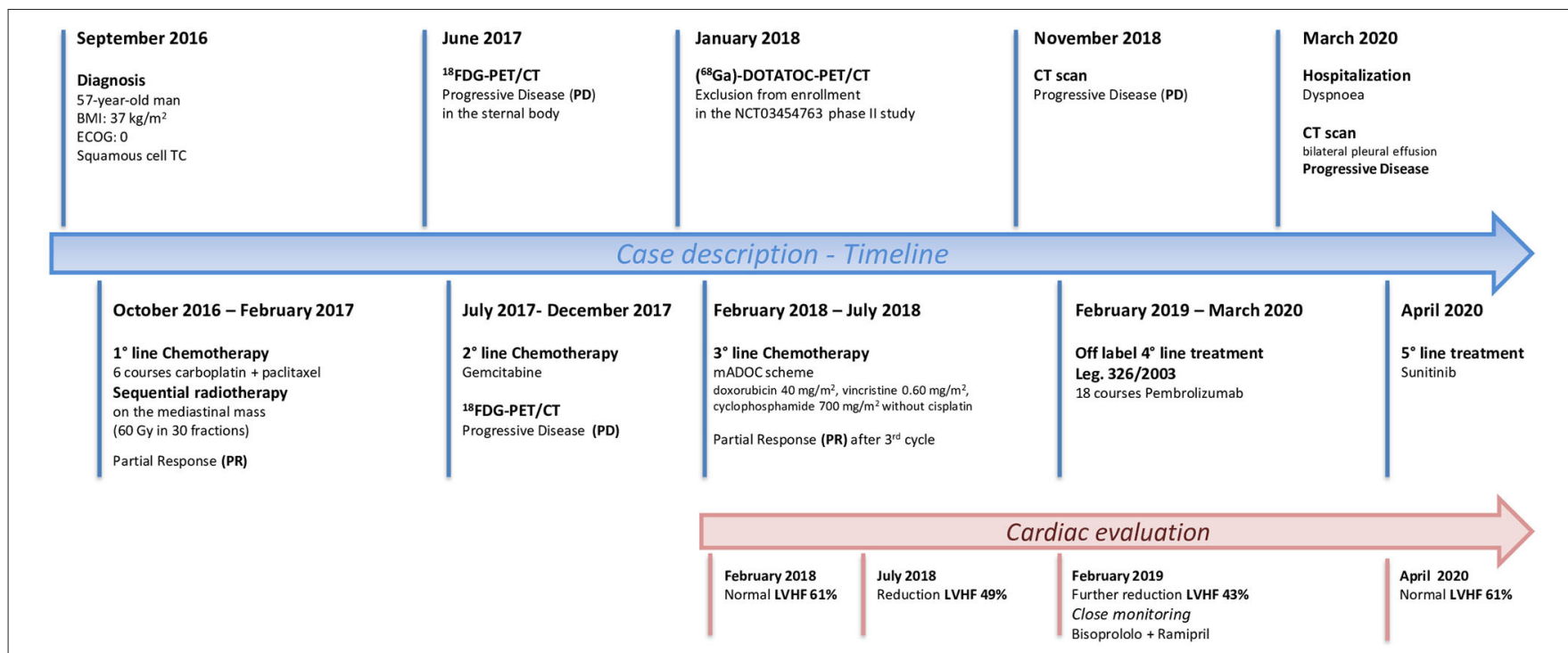

FIGURE 3 | Timeline showing patient history from the diagnosis of Squamous Cell Thymic Cancer to the most recent treatment perspectives.

TABLE 1 | Immunotherapies in thymic tumours.

\begin{tabular}{|c|c|c|c|c|c|c|c|c|c|}
\hline References & Patients & T; TC (SCTC) & Drug & ORR \% & DCR \% & mPFS & mos & $\begin{array}{c}\text { Cardiac } \\
\text { side-effect }\end{array}$ & Grade \\
\hline Giaccone et al. (18) & 40 & $0 ; 40$ (19) & Pembrolizumab & 22.5 & 75.5 & $\begin{array}{c}4.2 \mathrm{mo} \\
(8.9-10.3)\end{array}$ & $\begin{array}{c}24.9 \mathrm{mo} \\
(15.5-\mathrm{NR})\end{array}$ & $\begin{array}{c}5 \% \text { (2 patients- } \\
\text { myocarditis) }\end{array}$ & 4 \\
\hline Cho et al. (20) & 33 & 7; 26 (NRep) & Pembrolizumab & $\begin{array}{l}\text { T: } 28.6 \\
\text { TC: } 19.2\end{array}$ & $\begin{array}{l}\text { T: } 100 \\
\text { TC: } 73\end{array}$ & $6.1 \mathrm{mo}$ & - & $\begin{array}{c}9.1 \% \text { (3 patients- } \\
\text { myocarditis) }\end{array}$ & $\geq 3$ \\
\hline Katsuya et al. (26) & 15 & $0 ; 15$ (13) & Nivolumab & 0 & 73 & $\begin{array}{c}3.8 \mathrm{mo} \\
(1.9-5.6)\end{array}$ & NA (11.3-NA) & $0 \%$ & - \\
\hline
\end{tabular}

DCR, disease control rate; mo, months; mPFS, median progression-free survival; $m O S$, median overall survival; NR, not reached; ORR, objective response rate; pts, patients; T, thymoma; TC, thymic carcinoma; NRep, not reported; SCT, squamous cell thymic carcinoma; NA, not available.

observed and patient recruitment was stopped after a preplanned futility interim analysis. The DCR was $73 \%$ and the median PFS 3.8 mouths (26). Finally, data on the safety and activity of the anti PD-1 avelumab have recently been reported in patients with advanced thymoma treated with at least one prior standard therapy. Of the 7 patients with B1-B3 thymoma, 2 had confirmed PR, 2 unconfirmed PR, 2 SD, and one disease progression (27).

Results from different clinical trials have shown the promising activity of Immune checkpoint inhibitors (ICI) targeting the PD-1/PD-L1 axis in patients with advanced TC (Table 1) and NCCN guidelines recently added pembrolizumab as a potentially effective drug for second-line therapy in TC. Considerable activity has been noticed in subgroup of patients with high tumour PD-L1 levels.

Immune-related toxicity can affect potentially any organ, in particular the cardiovascular system (28). Myocarditis is a serious autoimmune condition. ICI-related myocarditis typically develops during the early stages of treatment (17-34 days after initiation of ICI therapy) and can show a rapid course, with severely reduced LV function, hemodynamic instability, and the need for intensive care (29). Although rarely described in the literature it has been reported in $5 \%$ of patients with TC and in $43-57 \%$ of patients with thymoma recruited in clinical trials and treated with ICIs (Table 1). Little is known about the underlying pathogenetic mechanisms of immune-related toxicity. Recent data suggests that the tumour itself may play an important role in mediating an immune response against cardiac structures due to epitopes shared between the myocardium and the tumour. In TETs, the incidence of myocarditis is higher in thymomas characterized by autoimmune disorders than in TCs (30).

In the case of our patient, literature data, tumour histology (squamous cell TC vs. thymoma), the previous therapeutic lines administered and the absence of autoimmune disorders prompted us to proceed with pembrolizumab, despite the unknown expression level of PD-L1 in tumour cells. Given the heavy pre-treatment and comorbidity of our patient, we obtained an excellent result. The PFS of more than 1 year is between 
2- and 3-fold longer than the median PFS reported in the aforementioned clinical trials on anti PD-1/PD-L1 antibodies. In addition, our patient did not experience any cardiac adverse effects. The results obtained also stress the importance of a multidisciplinary team approach, thanks to which a safer and more suitable therapeutic strategy was chosen for our patient. The treatment not only led to a good clinical result but has also enabled us to proceed with an additional therapeutic line (sunitinib) thanks to the recovery of normal cardiac function following the damage caused by previous chemoand radio-therapy.

\section{CONCLUSIONS}

We presented the case of a patient with squamous cell TC and multiple metastatic sites, unsuccessfully treated with three prior systemic therapy, in whom we obtained good disease control from the off label use of the anti PD-1 antibody pembrolizumab. Our findings and literature review suggest that anti PD1/PDL1 drugs could be a novel treatment for TC with promising activity and safety, despite the few data available from small monocentre trials. Further research on phase III multicentre

\section{REFERENCES}

1. Siesling S, van der Zwan JM, Izarzugaza I, Jaal J, Treasure T, Foschi R, et al. Rare thoracic cancer, including peritoneum mesothelioma. Eur J Cancer. (2012) 48:949-60. doi: 10.1016/j.ejca.2012.02.047

2. Ströbel P, Weis CA, Marx A. Thymic carcinomas. Pathologe. (2016) 37:42533. doi: 10.1007/s00292-016-0194-4

3. Ahamd U, Yao X, Detterbeck F, Huang J, Antonicelli A, Filosso PL, et al. Thymic carcinoma outcome and prognosis: results of an international analysis. J Thorac Cardiovasc Surg. (2015) 149:95-101. doi: 10.1016/j.jtcvs.2014.09.124

4. Ruffini E, Detterbeck F, Van Raemdonk D, Rocco G, Thomas P, Weder W, et al. Thymic carcinoma: a cohort study of patients from the European society of thoracic surgeons database. J Thorac Oncol. (2014) 9:5418. doi: 10.1097/JTO.0000000000000128

5. Zhao Y, Zhao H, Hu D, Fan L, Shi J, Fang W. Surgical treatment and prognosis of thymic squamous cell carcinoma: a retrospective analysis of 105 cases. Ann Thorac Surg. (2013) 96:1019-24. doi: 10.1016/j.athoracsur.2013.04.078

6. Wang CL, Gao LT, Lv CX, Zhu L, Fang WT. Outcome of nonsurgical treatment for locally advanced thymic tumors. J Thorac Dis. (2016) 8:70510. doi: $10.21037 /$ jtd.2016.01.79

7. Scorsetti M, Leo F, Trama A, D’Angelillo R, Serpico D, Macerelli M, et al. Thymoma and thymic carcinomas. Crit Rev Oncol Hematol. (2016) 99:33250. doi: 10.1016/j.critrevonc.2016.01.012

8. Kelly RJ, Petrini I, Rajan A, Wang Y, Giaccone G. Thymic malignances: from clinical management to target therapy. J Clin Oncol. (2011) 29:48207. doi: 10.1200/JCO.2011.36.0487

9. Girard N, Ruffini E, Marx A, Faivre-Finn C, Peters S, ESMO Guidelines Committee. Thymic epithelial tumours: ESMO clinical practice guidelines for diagnosis, treatment and follow-up. Ann Oncol. (2015) 26 (Suppl. 5):v4055. doi: 10.1093/annonc/mdv277

10. Lattanzio R, La Sorda R, Facciolo F, Sioletic S, Lauriola L, Martucci $\mathrm{R}$, et al. Thymic epithelial tumors express vascular endothelial growth factors and their receptors as potential targets of antiangiogenic therapy: a tissue micro array-based multicenter study. Lung Cancer. (2014) 85:1916. doi: 10.1016/j.lungcan.2014.05.010

11. Thomas A, Rajan A, Berman A, Tomita Y, Brzezniak C, Lee MJ, et al. Sunitinib in patients with chemotherapy-refractory thymoma and thymic clinical trials is warranted to investigate the efficacy and safety anti PD-1/PD-L1 drugs.

\section{ETHICS STATEMENT}

The studies involving human participants were reviewed and approved by Comitato Etico della Romagna - CEROM. The patients/participants provided their written informed consent to participate in this study. The authors obtained patient consent for publication of clinical data and images.

\section{AUTHOR CONTRIBUTIONS}

$\mathrm{AC}$ and $\mathrm{AB}$ drafted the manuscript and carried out the literature review. DO provided the images and the pathology review. TI supervised the study. CM and VD revised the manuscript for intellectual content. All authors read and approved the final version of the manuscript for submission.

\section{ACKNOWLEDGMENTS}

The authors thank Gráinne Tierney and Cristiano Verna for editorial assistance. carcinoma: an open-label phase 2 trial. Lancet Oncol. (2015) 16:17786. doi: 10.1016/S1470-2045(14)71181-7

12. Sato J, Satouchi M, Itoh S, Okuma $Y$, Niho S, Mizugaki $H$, et al. Lenvatinib in patients with advanced or metastatic thymic carcinoma (REMORA): a multicentre, phase 2 trial. Lancet Oncol. (2020) 21:84350. doi: 10.1016/S1470-2045(20)30162-5

13. Zucali PA, De Pas T, Palmieri G, Favaretto A, Chella A, Tiseo M, et al. Phase II study of everolimus in patients with thymoma and thymic carcinoma previously trated cisplatin-based chemotherapy. J Clin Oncol. (2018) 36:3429. doi: 10.1200/JCO.2017.74.4078

14. NCCN Clinical guidelines in Oncology. Thymomas and Thymic Carcinomas Version 2.2018. (2018). Available online at: https://www2.tri-kobe.org/nccn/ guideline/lung/english/thymic.pdf (accessed February 16, 2016).

15. Tateishi K, Ko R, Shukuya T, Okuma Y, Watanabe S, Kuyama S, et al. Clinical outcome of second-line chemotherapy in patients with previously treated advanced thymic carcinoma: a retrospective analysis of 191 patients from the NEJ023 study. Oncologist. (2020) 25:e66874. doi: 10.1634/theoncologist.2019-0593

16. Remon J, Passiglia F, Ahn MJ, Barlesi F, Forde PM, Garon EB, et al. Immune checkpoint inhibitors in thoracic malignancies: review of the existing evidence by an IASLC expert panel and recommendations. J Thorac Oncol. (2020) 15:914-47. doi: 10.1016/j.jtho.2020. 03.006

17. Morrot A. Cancer immunotherapy: lessons from the parasites to improve fighting against tumors. Precis Cancer Med. (2020) 3:11. doi: $10.21037 / \mathrm{pcm} .2020 .02 .02$

18. Giaccone G, Kim C, Thompson J, McGuire C, Kallakury B, Chahine JJ, et al. Pembrolizumab in patients with thymic carcinoma: a single arm, single centre, phase 2 study. Lancet Oncol. (2018) 19:347-55. doi: 10.1016/S1470-2045(18)30062-7

19. Thomas A, Shanbhag S, Haglund K, Berman A, Jakopovic M, Szabo E, et al. Characterization and management of cardiac involvement of thymic epithelial tumors. J Thorac Oncol. (2013) 8:246-9. doi: 10.1097/JTO.0b013e3182 7bd931

20. Cho J, Kim HS, Ku BM, Choi YL, Christescu R, Han J, et al. Pembrolizumab for patients with refractory or relapsed thymic epithelial tumor: an open label phase II trial. J Clin Oncol. (2019) 37:2162-70. doi: 10.1200/JCO.2017.7 7.3184 
21. Amin MB, Edge SB, Greene FL, Byrd DR, Brookland RK, Washington MK, et al. (eds.). AJCC Cancer Staging Manual. 8th ed. New York, NY: Springer (2017).

22. Eisenhauer EA, Therasse P, Bogaerts J, Schwartz LH, Sargent D, Ford R, et al. New response evaluation criteria in solid tumors: revised RECIST guideline (version 1.1). Eur J Cancer. (2009) 45:228-47. doi: 10.1016/j.ejca.2008. 10.026

23. Hamaoka T, Madewell JE, Podoloff DA, Hortobagyi GN, Ueno NT. Bone imaging in metastatic breast cancer. J Clin Oncol. (2004) 22:294253. doi: 10.1200/JCO.2004.08.181

24. Brown JA, Dorfman DM, Ma FR, Sullivan EL, Munoz O, Wood CR, et al. Blockade of programmed death-1 ligands on dendritic cells enhances T cell activation and cytokine production. J Immunol. (2003) 170:125766. doi: 10.4049/jimmunol.170.3.1257

25. Padda SK, Riess JW, Shwartz EJ, Tian L, Kohort HE, Neal JW, et al. Diffuse high intensity PD-L1 staining in thymic epithelial tumors. J Thorac Oncol. (2015) 10:500-8. doi: 10.1097/JTO.0000000000000429

26. Katsuya Y, Hourinouchi H, Seto T, Umemura S, Hosomi Y, Satouchi M, et al. Single arm multicentre, phase II trial of nivolumab for unresecable or recurrent thymic carcinoma: PRIMER study. Eur J Cancer. (2019) 113:7886. doi: 10.1016/j.ejca.2019.03.012

27. Rajan A, Heery CR, Thomas A, Mammen AL, Perry S, O'Sullivan Coyne G, et al. Efficacy and tolerability of anti-programmed death ligand 1 (PD-L1) antibody (avelumab) treatment in advance thymoma. J Immunother Cancer. (2019) 7:269. doi: 10.1186/s40425-019-0723-9

28. June $\mathrm{CH}$, Warshauer JT, Bluestone JA. Is autoimmunity the achilles' heel of cancer immunotherapy? Nat Med. (2017) 23:540-7. doi: 10.1038/nm.4321

29. Escudier M, Cautela J, Malissen N, Ancedy Y, Orabona M, Pinto $\mathrm{J}$, et al. Clinical features, management, and outcomes of immune checkpoint inhibitor-related cardiotoxicity. Circulation. (2017)136:20857. doi: 10.1161/CIRCULATIONAHA.117.030571

30. Johnson DB, Balko JM, Compton ML, Chalkias S, Gorham J, Xu Y. Fulminant myocarditis with combination immune checkpoint blockade. N Engl J Med. (2016) 375:1749-55. doi: 10.1056/NEJMoa1609214

Conflict of Interest: The authors declare that the research was conducted in the absence of any commercial or financial relationships that could be construed as a potential conflict of interest.

Copyright (C) 2020 Cafaro, Bongiovanni, Di Iorio, Oboldi, Masini and Ibrahim. This is an open-access article distributed under the terms of the Creative Commons Attribution License (CC BY). The use, distribution or reproduction in other forums is permitted, provided the original author(s) and the copyright owner(s) are credited and that the original publication in this journal is cited, in accordance with accepted academic practice. No use, distribution or reproduction is permitted which does not comply with these terms. 